\title{
Variants in RETN gene are associated with steroid-induced osteonecrosis of the femoral head risk among Han Chinese people
}

Feimeng An ${ }^{1,2+}$, Litian Zhang ${ }^{1,2+}$, Hongyan Gao ${ }^{1,2}$, Jiaqi Wang ${ }^{1,2}$, Chang Liu², ${ }^{1,2}$ Ye Tian ${ }^{1,2}$, Chao Ma ${ }^{1,2}$, Jian Zhao ${ }^{1,2}$, Kunzheng Wang ${ }^{3^{*}}$ and Jianzhong Wang ${ }^{2^{*}}$ (D)

\begin{abstract}
Background: Gene polymorphism has an important influence on RETN gene expression level, and the increased level of resistin encoded in RETN will lead to metabolic disorder, especially lipid metabolism. Moreover, steroidinduced osteonecrosis of the femoral head (steroid-induced ONFH) is closely related to lipid metabolism level, so this study is intended to explore the relationship of RETN polymorphisms with susceptibility to steroid-induced ONFH in the Chinese Han population.
\end{abstract}

Methods: In this case-control study, eight single-nucleotide polymorphisms (SNPs) of RETN were genotyped by the Agena MassARRAY system in 199 steroid-induced ONFH patients and 200 healthy controls. The relationship between RETN polymorphisms and steroid-induced ONFH risk was assessed using genetic models and haplotype analyses. Odds ratio (OR) and 95\% confidence intervals (Cls) were obtained by logistic regression adjusted for age.

Results: We found significant differences in the distribution of HDL-C, TG/HDL-C, and LDL-C/HDL-C between the patients and the control group $(p<0.05)$. In allele model and genotype model analysis, rs34861192, rs3219175, rs3745368, and rs 1477341 could reduce the risk of steroid-induced ONFH. Further stratified analysis showed that rs3745367 was related to the clinical stage of patients, and rs1477341 was significantly correlated with an increased TG level and a decreased TC/HDL-C level. The linkage analysis showed that two SNPs (rs34861192 and rs3219175) in RETN even significant linkage disequilibrium.

Conclusions: Our results provide the firstly evidence that RETN gene polymorphisms were associated with a reduced risk of steroid-induced ONFH in Chinese Han population.

Keywords: Steroid-induced osteonecrosis of the femoral head (steroid-induced ONFH), Resistin (RETN), Genetic polymorphisms, Case-control study

\footnotetext{
*Correspondence: 2437935260@qq.com; Wangjianzhong1111@163.com

${ }^{\dagger}$ Feimeng An and Litian Zhang are co-first authors.

${ }^{3}$ The Second Affiliated Hospital of Xi'an Jiaotong University, \#157 Xi Wu Road, Xi'an 710004, Shaanxi Province, China

${ }^{2}$ Department of Trauma Orthopedics, The Second Affiliated Hospital of Inner

Mongolia Medical University, Hohhot 010030, Inner Mongolia, China

Full list of author information is available at the end of the article
}

(C) The Author(s). 2020 Open Access This article is distributed under the terms of the Creative Commons Attribution 4.0 International License (http://creativecommons.org/licenses/by/4.0/), which permits unrestricted use, distribution, and reproduction in any medium, provided you give appropriate credit to the original author(s) and the source, provide a link to the Creative Commons license, and indicate if changes were made. The Creative Commons Public Domain Dedication waiver (http://creativecommons.org/publicdomain/zero/1.0/) applies to the data made available in this article, unless otherwise stated. 


\section{Background}

With the wide application of glucocorticoid in the treatment of rheumatic diseases, autoimmune diseases, hematopoietic system diseases, and other diseases, steroid-induced osteonecrosis of the femoral head (steroid-induced ONFH) has become the most common non-traumatic osteonecrosis of the femoral head $(\mathrm{ONFH})$ type in clinical practice $[1,2]$. Epidemiological studies in East Asia have shown that $47.4 \%$ of all cases diagnosed as non-traumatic ONFH arose as a direct result of steroids [3]. As a degenerative bone disease, it can cause the femoral head to collapse, which subsequently damage the hip joint and seriously reduces the patients quality of life and is difficult to reverse [3, 4]. However, it is challenging to fully elucidate the pathogenesis of steroid-induced ONFH due to the various effects of steroids on multiple systems involved in osteoblast differentiation, osteoblast and osteoclast apoptosis, lipid metabolism, calcium metabolism, and coagulation [5].

Previous studies have demonstrated that only a subset of patients develops ONFH within a few weeks of hormone therapy, suggesting that genetic factors may determine susceptibility to steroid-induced ONFH [6]. Single-nucleotide polymorphisms (SNPs) are the most frequent variation that occurs in a single nucleotide at a specific position in the genome. Numerous SNPs have been identified through sequencing, and many of them in critical genes such as MMP-8 [1], MMP-9 [7], MMP-14 [8], ABCB1 [9], and VEGFA [10] were demonstrated to be associated with steroid-induced ONFH susceptibility.

RETN, also known as ADSF and FIZZ3, is located on chromosome 19p13. RETN gene encodes resistin, which is a hormone secreted by fat cells and belongs to the cysteine-rich $\mathrm{C}$-terminal domain proteins called resistinlike molecules [11]. It was first observed in adipocytes, then in monocytes, macrophages, and the spleen, and more importantly, in the human sebaceous glands and cultured sebaceous glands [12]. Some studies have demonstrated that elevated levels of resistin lead to metabolic disorders, which are associated with diabetes, non-insulin dependent, acquired systemic lipodystrophy, rheumatoid arthritis, etc. [13-15]. Most importantly, it is the product of the secretion of fat cells and its related pathways include lipogenesis [16]. Elevated levels of resistin may contribute to lipid metabolism disorders [15], which can lead to steroid-induced ONFH. Thus RETN may induce steroid-induced ONFH by affecting lipid metabolism.

To our knowledge, no previous studies have investigated the relationship between steroid-induced ONFH risk and RETN polymorphisms. Therefore, we conduct a case-control study to evaluate the possible relationship of RETN gene polymorphisms at allele, genotype, and haplotype interface with the development of steroid-induced ONFH among Chinese Han population.

\section{Materials and methods Study participants}

The present hospital-based case-control study recruited 199 patients diagnosed with steroid-induced ONFH and 200 unrelated control subjects at the orthopedic hospital of Inner Mongolia Medical University (Inner Mongolia, China) from 2016 to 2019. These individuals were informed of the purpose of the study and signed informed consent.

We collected epidemiological information from standardized questionnaires and collected clinical information from medical records and imaging examinations to finally determine the case and control individuals. Patients with steroid-induced ONFH in this study had common clinical manifestations of hip pain, lower limb muscular atrophy, and joint dysfunction and were also receiving long-term steroid intake of more than $16 \mathrm{mg}$ per day or high-dose impulsive treatment with steroids lasting more than 1 week prior to the onset of these symptoms [1]. An $\mathrm{X}$-ray examination and additional magnetic resonance imaging (MRI) and bone scan analyses were performed on each patient as necessary. We also used strict exclusion criteria to exclude the following patients: patients with traumatic ONFH and other hip diseases; patients who consumed more than $400 \mathrm{ml}$ of alcohol per week; patients with a history of severe disease or severe chronic disease, such as renal insufficiency, diabetes, and cardiovascular and cerebrovascular diseases. The included controls met the following criteria: they had no hip pain, anteroposterior and frog-leg lateral pelvic radiographs did not show any lesions, no history of thromboembolic events, no symptoms of hip disease, and no chronic diseases such as renal insufficiency, diabetes, and cardiovascular and cerebrovascular diseases.

\section{SNPs selection and genotyping}

Individual demographics information and the clinical characteristics of patients were collected by well-trained interviewers. Subsequently, $5 \mathrm{~mL}$ of peripheral blood from each participant was collected by a specialized technician and stored into tubes containing ethylenediaminetetraacetic acid (EDTA). After centrifugation, the specimens were stored at $80^{\circ} \mathrm{C}$ until further analysis. All volunteers signed an informed consent form explaining the research 
purpose of our study. Genomic DNA was isolated from peripheral whole blood employing the Gold Mag-Mini whole blood genomic DNA purification kit (Gold Mag Co. Ltd., Xi'an, China) following the manufacturer's instructions and quantified by a Nano Drop spectrophotometer 2000 C (Thermo Scientific, Waltham, MA, USA).

In this study, eight SNPs (rs7408174, rs34861192, rs34124816, rs3219175, rs3745367, rs3745368, rs3745369, rs1477341) were selected for genotyping. These SNPs had minor allele frequencies $>5 \%$ in the 1000 Genome Projects (http://www.internationalgenome.org/). Polymerase chain reaction (PCR) extension primers were designed for these SNPs by the MassARRAY Assay Design 3.0 software (Agena). SNP genotyping analysis was carried out at an Agena MassARRAY RS1000 Instrument (Shanghai, China) system according to the standard scheme recommended by the manufacturer, and data were managed and analyzed by the Agena Typer 4.0 software [17]. In addition, about $10 \%$ of the total samples were randomly selected to repeat genotyping, and the reproducibility was $100 \%$.

\section{Statistical analyses}

All of the statistical analyses were performed with the SPSS statistical package, version 19.0 (SPSS Inc., Chicago, IL, USA). Hardy-Weinberg equilibrium (HWE) of each SNP in the control group was tested by Fisher's exact test. Allele frequencies and genotype frequencies for each SNP of case and control subjects were evaluated using the chi-squared test. Odds ratios (ORs) and 95\% confidence intervals (CIs) were determined using logistic regression analysis with adjustments for age and gender. The wildtype allele was used as a reference. Multiple genetic model analyses (codominant, dominant, recessive, and log-additive) were applied using the PLINK software (http://pngu.mgh.harvard.edu/purcell/plink/) to assess the association between SNPs and steroidinduced ONFH. Finally, the Haploview software package (version 4.2) and the SHEsis software (http://analysis.bio-x.cn/myAnalysis.php) were used to analyze the pairwise linkage disequilibrium (LD), haplotype construction, and genetic association of polymorphisms. All $p$ values in this study were twosided, and $p$ value of less than 0.05 was the cutoff value for statistical significance.

\section{Results}

The basic characteristics of study subjects

The clinical information and the demographic characteristics of both the cases and controls were shown in Table 1. No significant difference was observed
Table 1 Characteristics of the participants

\begin{tabular}{|c|c|c|c|}
\hline Variables & Cases $(n=199)$ & Controls $(n=200)$ & $p$ value \\
\hline$\overline{\operatorname{Sex} N}$ & & & $0.966^{\mathrm{b}}$ \\
\hline Male & 115 & 116 & \\
\hline Female & 84 & 84 & \\
\hline Age, years (mean $\pm S D$ ) & $41.15 \pm 12.934$ & $41.20 \pm 8.662$ & $0.961^{a}$ \\
\hline \multicolumn{4}{|l|}{ Clinical stages } \\
\hline Stage II & 47 & & \\
\hline Stage III & 94 & & \\
\hline Stage IV & 58 & & \\
\hline \multicolumn{4}{|l|}{ Hip lesions } \\
\hline unilateral & 59 & & \\
\hline bilateral & 140 & & \\
\hline $\mathrm{TC}(\mathrm{mmol} / \mathrm{L})$ & $4.50 \pm 0.923$ & $4.54 \pm 0.814$ & 0.703 \\
\hline $\mathrm{TG}(\mathrm{mmol} / \mathrm{L})$ & $1.84 \pm 1.455$ & $1.76 \pm 1.076$ & 0.569 \\
\hline $\mathrm{HDL}-\mathrm{C}(\mathrm{mmol} / \mathrm{L})$ & $1.07 \pm 0.269$ & $1.15 \pm 0.214$ & $0.000^{\mathrm{a}}$ \\
\hline $\mathrm{LDL}-\mathrm{C}(\mathrm{mmol} / \mathrm{L})$ & $2.64 \pm 0.765$ & $2.56 \pm 0.728$ & 0.285 \\
\hline $\mathrm{TC} / \mathrm{HDL}-\mathrm{C}$ & $4.41 \pm 1.178$ & $4.04 \pm 0.856$ & $0.000^{\mathrm{a}}$ \\
\hline $\mathrm{TG} / \mathrm{HDL}-\mathrm{C}$ & $1.94 \pm 1.930$ & $1.64 \pm 1.149$ & 0.059 \\
\hline LDL-C/HDL-C & $2.58 \pm 0.821$ & $2.30 \pm 0.767$ & $0.001^{\mathrm{a}}$ \\
\hline
\end{tabular}

TC total cholesterol, TG triglycerides, LDL-C low-density lipoprotein cholesterol, $L D L-C$ high-density lipoprotein cholesterol

${ }^{a} p$ value was calculated by independent samples $t$ test

${ }^{\mathrm{b}} p$ value was calculated by chi-squared test

$p<0.05$ indicates statistical significance

between cases and controls in regard to age, gender, TC, TG, LDL-C, and TG/HDL-C. However, there were significant differences in the distribution of HDL-C, TG/HDL-C, and LDL-C/HDL-C between the two groups $(p<0.05)$.

\section{The associations between RETN SNPs and steroid-induced ONFH}

The basic information of the selected SNPs are presented in Table 2. Allele frequencies of all loci in controls were in accordance with Hardy-Weinberg equilibrium $(p>0.05)$. As indicated by the italicized $p$ values in Table 2 , the chi-squared test was used to assess the risk of genetic polymorphisms in the allele model, and it was found that $\mathrm{rs} 34861192(\mathrm{OR}=1.64$, 95\% CI $=0.44-0.93, p=0.019), \quad r s 3219175 \quad(\mathrm{OR}=0.61$, $95 \% \mathrm{CI}=0.42-0.89, p=0.010), \quad \mathrm{rs} 3745368 \quad(\mathrm{OR}=0.62$, $95 \% \mathrm{CI}=0.41-0.93, p=0.020), \quad \mathrm{rs} 1477341 \quad(\mathrm{OR}=0.74$, $95 \% \mathrm{CI}=0.56-0.98, p=0.036)$ were related to a reduced risk of steroid-induced ONFH.

\section{Associations between genotype frequencies and steroid- induced ONFH risk}

As presented in Table 3, we further analyzed the relationship between genotype frequency and steroidinduced ONFH risk through multiple genetic 
Table 2 Basic information of candidate SNPS of RETN gene in this study

\begin{tabular}{|c|c|c|c|c|c|c|c|c|}
\hline \multirow[t]{2}{*}{ SNP ID } & \multirow[t]{2}{*}{ Gene } & \multirow[t]{2}{*}{ Band } & \multirow{2}{*}{$\begin{array}{l}\text { Alleles } \\
\text { A/B }\end{array}$} & \multicolumn{2}{|l|}{ MAF } & \multirow{2}{*}{$\begin{array}{l}\text { HWE- } \\
p^{\mathrm{a}}\end{array}$} & \multirow[t]{2}{*}{ OR $(95 \% \mathrm{Cl})$} & \multirow{2}{*}{$\begin{array}{l}p^{\mathrm{b}} \\
\text { value }\end{array}$} \\
\hline & & & & Case & $\overline{\text { Control }}$ & & & \\
\hline rs7408174 & RETN & 19 & $C / T$ & 0.265 & 0.233 & 0.556 & $1.19(0.86-1.64)$ & 0.287 \\
\hline rs34861192 & RETN & 19 & $A / G$ & 0.136 & 0.198 & 0.654 & $0.64(0.44-0.93)$ & $0.019^{*}$ \\
\hline rs34124816 & RETN & 19 & $\mathrm{~A} / \mathrm{C}$ & 0.075 & 0.100 & 1.000 & $0.73(0.45-1.20)$ & 0.219 \\
\hline rs3219175 & RETN & 19 & $A / G$ & 0.132 & 0.200 & 0.659 & $0.61(0.42-0.89)$ & $0.010^{*}$ \\
\hline rs3745367 & RETN & 19 & $A / G$ & 0.324 & 0.390 & 0.882 & $0.75(0.56-1.00)$ & 0.052 \\
\hline rs3745368 & RETN & 19 & $\mathrm{~A} / \mathrm{G}$ & 0.113 & 0.171 & 1.000 & $0.62(0.41-0.93)$ & $0.020^{*}$ \\
\hline rs3745369 & RETN & 19 & $C / G$ & 0.303 & 0.337 & 0.636 & $0.85(0.63-1.15)$ & 0.305 \\
\hline rs1477341 & RETN & 19 & $\mathrm{~A} / \mathrm{T}$ & 0.405 & 0.480 & 0.479 & $0.74(0.56-0.98)$ & $0.036^{*}$ \\
\hline
\end{tabular}

SNP single-nucleotide polymorphism, HWE Hardy-Weinberg equilibrium, OR odds ratio, 95\% Cl 95\% confidence interval, $M A F$ minor allele frequency $p^{\mathrm{a}}$ and $p^{\mathrm{b}}$ were calculated by chi-squared test

Italicized values are statistically significant

${ }^{*} p<0.05$ indicates statistical significance

models. We found that rs34861192 was associated with a lower steroid-induced ONFH risk in codominant (AA vs GG: $\mathrm{OR}=0.10,95 \% \mathrm{CI}=0.01-$ $0.79, p=0.0329$ ), recessive (AA vs GG-AG: $\mathrm{OR}=$ $0.11,95 \% \mathrm{CI}=0.01-0.85, p=0.035)$, and log-additive models $(\mathrm{OR}=0.63,95 \% \mathrm{CI}=0.43-0.93, p=0.019)$. Rs3219175 was identified to decrease the steroidinduced ONFH risk in codominant (AA vs GG: $\mathrm{OR}=0.10,95 \% \mathrm{CI}=0.01-0.79, p=0.029)$, dominant (AG-AA vs $\mathrm{GG}$ : $\mathrm{OR}=0.63,95 \% \mathrm{CI}=0.41-0.98, p=$ 0.038 ), recessive (AA vs GG-AG: $\mathrm{OR}=0.11,95 \%$ $\mathrm{CI}=0.01-0.86, p=0.0360)$, and log-additive models $(\mathrm{OR}=0.60,95 \% \mathrm{CI}=0.41-0.88, p=0.010) . \mathrm{Rs} 3745368$ was also related to decreasing steroid-induced ONFH risk in dominant (AG-AA vs GG: OR $=0.61,95 \%$ $\mathrm{CI}=0.38-0.95, p=0.030)$, and log-additive models (A vs $\mathrm{G}:$ OR $=0.61,95 \% \mathrm{CI}=0.41-0.92, p=0.019$ ). Rs1477341 also showed an association with the steroid-induced ONFH risk in dominant (AT-AA vs TT: $\quad \mathrm{OR}=0.62,95 \% \mathrm{CI}=0.40-0.96, \quad p=0.03)$ and log-additive models $(\mathrm{OR}=0.73,95 \% \mathrm{CI}=0.55-0.98$, $p=0.036)$.

\section{Relationship between RETN SNPs and clinical features of steroid-induced ONFH}

We also explored the relationship between the RETN SNPs and steroid-induced ONFH clinical features, including gender, hip lesions, clinical stages, and the expression level of lipid. We observed that rs3745367 shows association with the clinical stages in Table 4 $(p=0.049)$. In terms of lipid metabolism level of patients, it was found that compared with AA genotype carriers, HDL-C level of TT genotype carriers in rs1477341 was significantly higher, and TC level was lower. Meanwhile, the TC/HDL-C ratio of TT genotype carriers was significantly lower than that of AA genotype carriers (Table 5).

\section{Associations between haplotype analyses and steroid- induced ONFH risk}

The linkage analysis showed that two SNPs (rs34861192 and rs3219175) in RETN exhibited significant linkage disequilibrium (Fig. 1).

\section{GTEx database analysis}

Through GTEx database analysis, rs34861192 and rs3219175 ( $p=4.00 \mathrm{E}-14, p=1.60 \mathrm{E}-14)$ sites were observed to be associated with a reduced expression of RETN gene in whole blood.

\section{Discussion}

It is well-known that genetic studies have provided insights into many diseases, including steroid-induced ONFH. This study was designed to investigate the contribution of genetic variation in RETN to steroidinduced ONFH risk in a Chinese Han population. Allele, genotype, and haplotype frequencies of eight SNPs in the RETN gene between steroid-induced ONFH patients and healthy controls were compared, and stratification analyses were conducted. Our study found that the rs34861192, rs3219175, rs3745368, and rs1477341 were linked to a reduced risk of steroidinduced ONFH. To our knowledge, this is the first research to clarify the correlation between RETN gene variants with the risk of steroid-induced ONFH in Chinese Han population.

The RTEN gene encodes a resistin, which is also considered to be a biomarker or mediator of metabolic and inflammatory diseases [18, 19]. Some studies have shown that elevated levels of serum resistin lead to metabolic disorders, including obesity, insulin resistance, type 2 diabetes, hypertension, dyslipidemia, and atherosclerotic cardiovascular disease [20-22]. Moreover, it has been shown that the plasma resistin concentration was largely determined by polymorphisms 
Table 3 Analysis of the association between SNPs of RETN gene and steroid-induced ONFH risk

\begin{tabular}{|c|c|c|c|c|c|c|}
\hline SNP ID & Model & Genotype & Control & Case & OR $(95 \% \mathrm{Cl})$ & $p$ value \\
\hline \multirow[t]{8}{*}{ rs34861192 } & \multirow[t]{3}{*}{ Codominant } & GG & 130 & 146 & 1 & \\
\hline & & $A G$ & 61 & 52 & $0.76(0.49-1.18)$ & 0.220 \\
\hline & & AA & 9 & 1 & $0.10(0.01-0.79)$ & $0.029^{*}$ \\
\hline & \multirow[t]{2}{*}{ Dominant } & GG & 130 & 146 & 1 & \\
\hline & & AG-AA & 70 & 53 & $0.67(0.44-1.03)$ & 0.071 \\
\hline & \multirow[t]{2}{*}{ Recessive } & GG-AG & 191 & 198 & 1 & \\
\hline & & $\mathrm{AA}$ & 9 & 1 & $0.11(0.01-0.85)$ & $0.035^{*}$ \\
\hline & Log-additive & - & - & - & $0.63(0.43-0.93)$ & $0.019^{*}$ \\
\hline \multirow[t]{8}{*}{ rs3219175 } & \multirow[t]{3}{*}{ Codominant } & GG & 129 & 146 & 1 & \\
\hline & & $A G$ & 62 & 50 & $0.71(0.46-1.11)$ & 0.133 \\
\hline & & $\mathrm{AA}$ & 9 & 1 & $0.10(0.01-0.79)$ & $0.029^{*}$ \\
\hline & \multirow[t]{2}{*}{ Dominant } & GG & 129 & 146 & 1 & \\
\hline & & AG-AA & 71 & 51 & $0.63(0.41-0.98)$ & $0.038^{*}$ \\
\hline & \multirow[t]{2}{*}{ Recessive } & GG-AG & 191 & 196 & 1 & \\
\hline & & $\mathrm{AA}$ & 9 & 1 & $0.11(0.01-0.86)$ & $0.036^{*}$ \\
\hline & Log-additive & - & - & - & $0.60(0.41-0.88)$ & $0.010^{*}$ \\
\hline \multirow[t]{8}{*}{ rs3745368 } & \multirow[t]{3}{*}{ Codominant } & GG & 137 & 156 & 1 & \\
\hline & & $A G$ & 56 & 41 & $0.64(0.40-1.02)$ & 0.059 \\
\hline & & AA & 6 & 2 & $0.29(0.06-1.45)$ & 0.131 \\
\hline & \multirow[t]{2}{*}{ Dominant } & GG & 137 & 156 & 1 & \\
\hline & & AG-AA & 62 & 43 & $0.61(0.38-0.95)$ & $0.030^{*}$ \\
\hline & \multirow[t]{2}{*}{ Recessive } & GG-AG & 193 & 197 & 1 & \\
\hline & & $\mathrm{AA}$ & 6 & 2 & $0.32(0.06-1.63)$ & 0.170 \\
\hline & Log-additive & - & - & - & $0.61(0.41-0.92)$ & $0.019^{*}$ \\
\hline \multirow[t]{8}{*}{ rs1477341 } & \multirow[t]{3}{*}{ Codominant } & $\pi$ & 51 & 68 & 1 & \\
\hline & & AT & 105 & 90 & $0.64(0.41-1.02)$ & 0.059 \\
\hline & & AA & 43 & 32 & $0.56(0.31-1.00)$ & 0.051 \\
\hline & \multirow[t]{2}{*}{ Dominant } & $\pi$ & 51 & 68 & 1 & \\
\hline & & AT-AA & 148 & 122 & $0.62(0.40-0.96)$ & $0.030^{*}$ \\
\hline & \multirow[t]{2}{*}{ Recessive } & TT-AT & 156 & 158 & 1 & \\
\hline & & $\mathrm{AA}$ & 43 & 32 & $0.74(0.44-1.23)$ & 0.239 \\
\hline & Log-additive & - & - & - & $0.73(0.55-0.98)$ & $0.036^{*}$ \\
\hline
\end{tabular}

SNP single-nucleotide polymorphism, OR odds ratio, $95 \% \mathrm{Cl}$ 95\% confidence interval

$p$ value adjusted for age was calculated by logistic regression

Italicized values are statistically significant

${ }^{*} p<0.05$ indicates statistical significance

of RETN. For example, one study showed that plasma resistin was significantly correlated with rs34861192, rs34124816, rs3219175, rs3745367, and rs3745369 in RETN [23]. The study of Asano et al. found that rs34861192 and rs3745368 polymorphisms of RETN as robust and independent determinants of plasma resistin concentration [24]. An epigenome-wide association study suggested that rs34861192 and rs3219175 in the RETN promoter region may influence circulating resistin levels by affecting DNAm at cg02346997 and the abundance of
RETN mRNA in monocytes [3]. Besides, the minor (A) allele of rs34861192 was also found to be associated with a lower plasma resistin level $\left(R^{2}=0.010\right)$ [24]. The GG genotype carriers of RETN rs3219175 and rs3481192 exhibited higher levels of log-resistin than A allele carriers [25]. After multiple testing corrections, the rs1477341 also had a strong correlation with resistin level [26]. In our study, we found that the frequency of the minor (A) allele of rs34861192, rs3219175, rs3745368, and rs1477341 was 0.136, 0.132, 0.113, and 0.405, respectively. All four SNPs 
Table 4 The association of genotypes in RETN genes with the clinical phenotypes

\begin{tabular}{|c|c|c|c|c|c|c|c|c|c|c|c|}
\hline \multirow[t]{2}{*}{ SNP } & \multirow[t]{2}{*}{ Genotype } & \multicolumn{2}{|c|}{ Gender } & \multirow[t]{2}{*}{$p$} & \multicolumn{2}{|l|}{ Hip lesions } & \multirow[t]{2}{*}{$p$} & \multicolumn{3}{|c|}{ Clinical stages } & \multirow[t]{2}{*}{$p$} \\
\hline & & Male & Female & & Unilateral & Bilateral & & Stage II & Stage III & Stage IV & \\
\hline \multirow[t]{3}{*}{ rs7408174 } & CC & 8 & 6 & \multirow[t]{3}{*}{0.775} & 5 & 9 & \multirow[t]{3}{*}{0.285} & 3 & 5 & 6 & \multirow[t]{3}{*}{0.694} \\
\hline & $\mathrm{CT}$ & 42 & 35 & & 18 & 59 & & 19 & 39 & 19 & \\
\hline & $\Pi$ & 64 & 43 & & 36 & 71 & & 24 & 50 & 33 & \\
\hline \multirow[t]{3}{*}{ rs34861192 } & AA & 0 & 1 & \multirow[t]{3}{*}{0.465} & 0 & 1 & \multirow[t]{3}{*}{0.547} & 0 & 1 & 0 & \multirow[t]{3}{*}{0.193} \\
\hline & $A G$ & 29 & 23 & & 18 & 34 & & 15 & 28 & 9 & \\
\hline & GG & 86 & 60 & & 41 & 105 & & 32 & 65 & 49 & \\
\hline \multirow[t]{3}{*}{ rs34124816 } & $\mathrm{AA}$ & 94 & 77 & \multirow[t]{3}{*}{0.105} & 51 & 120 & \multirow[t]{3}{*}{0.650} & 41 & 78 & 52 & \multirow[t]{3}{*}{0.557} \\
\hline & CA & 20 & 6 & & 8 & 18 & & 6 & 14 & 6 & \\
\hline & $\mathrm{CC}$ & 1 & 1 & & 0 & 2 & & 0 & 2 & 0 & \\
\hline \multirow[t]{3}{*}{ rs3219175 } & AA & 0 & 1 & \multirow[t]{3}{*}{0.470} & 0 & 1 & \multirow[t]{3}{*}{0.592} & 0 & 1 & 0 & \multirow[t]{3}{*}{0.216} \\
\hline & $A G$ & 28 & 22 & & 17 & 33 & & 15 & 26 & 9 & \\
\hline & GG & 86 & 60 & & 41 & 105 & & 31 & 66 & 49 & \\
\hline \multirow[t]{3}{*}{ rs3745367 } & AA & 12 & 9 & \multirow[t]{3}{*}{0.775} & 3 & 18 & \multirow[t]{3}{*}{0.263} & 3 & 14 & 4 & \multirow[t]{3}{*}{$0.049^{*}$} \\
\hline & $A G$ & 48 & 39 & & 27 & 60 & & 24 & 44 & 19 & \\
\hline & GG & 55 & 36 & & 29 & 62 & & 20 & 36 & 35 & \\
\hline \multirow[t]{3}{*}{ rs3745368 } & $\mathrm{AA}$ & 1 & 1 & \multirow[t]{3}{*}{0.308} & 0 & 2 & 0.650 & 0 & 2 & 0 & 0.613 \\
\hline & $A G$ & 28 & 13 & & 12 & 29 & & 9 & 21 & 11 & \\
\hline & GG & 86 & 70 & & 47 & 109 & & 38 & 71 & 47 & \\
\hline rs3745369 & CC & 8 & 6 & 0.876 & 4 & 10 & 0.059 & 2 & 11 & 1 & 0.161 \\
\hline & $C G$ & 50 & 40 & & 20 & 70 & & 20 & 41 & 29 & \\
\hline & GG & 54 & 37 & & 35 & 56 & & 24 & 41 & 26 & \\
\hline rs1477341 & $\mathrm{AA}$ & 24 & 8 & 0.063 & 9 & 23 & 0.809 & 6 & 21 & 5 & 0.090 \\
\hline & AT & 48 & 42 & & 25 & 65 & & 21 & 43 & 26 & \\
\hline & $\pi$ & 35 & 33 & & 22 & 46 & & 17 & 25 & 26 & \\
\hline
\end{tabular}

$p$ values were calculated by logistic regression

Italicized value is statistically significant

${ }^{*} p<0.05$ indicates statistical significance

were linked to a lower risk of steroid-induced ONFH. Therefore, we speculated that these SNPs may reduce the occurrence of metabolic disorder by inducing a lower level of resistin, ultimately reducing the risk of steroid-induced ONFH.

Moreover, the plasma resistin levels have been shown to be related to serum concentrations of HDLcholesterol and triacylglycerol, IRI, and BMI [24]. In our study, we found that rs1477341 was significantly correlated with elevated HDL-C levels, and the HDLC level of rs1477341-TT carriers was significantly higher than that of rs1477341-AA carriers. Besides, our study also found that rs1477341 can reduce the risk of steroid-induced ONFH. These results further suggested the correlation between rs1477341 and resistin levels, HDL-C levels, metabolic disorders, and osteonecrosis. As for linkage disequilibrium analysis, it was also found that rs34861192 and rs3219175 were all located in the same LD segment, and both of them have been reported to be closely related to circulating resistin levels [27]. Similarly, our study also found that rs34861192 and rs3219175 were located in the same LD block.

Of course, our research has some limitations. Firstly, the inherent selecting and information bias were the unavoidable problems because this is a hospital-based, single-center study. Secondly, the number of cases in our study is not large enough to rule out false-negative results, so a larger sample size is needed for further confirmation. Third, our current study is a foundation's case-control study that requires further functional studies to understand the underlying genetic mechanism of steroid-induced ONFH. Despite the limitations noted above, our current findings provide scientific evidence for future studies on the effect of RETN on steroidinduced ONFH risk. 
Table 5 The association of genotypes in RETN genes with the clinical phenotypes

\begin{tabular}{|c|c|c|c|c|c|c|c|c|}
\hline SNP & Genotype & $\mathrm{TC}(\mathrm{mmol} / \mathrm{L})$ & TG (mmol/L) & $\mathrm{HDL}-\mathrm{C}(\mathrm{mmol} / \mathrm{L})$ & $\mathrm{LDL}-\mathrm{C}(\mathrm{mmol} / \mathrm{L})$ & $\mathrm{TC} / \mathrm{HDL}-\mathrm{C}$ & $\mathrm{TG} / \mathrm{HDL}-\mathrm{C}$ & LDL-C/HDL-C \\
\hline \multirow[t]{4}{*}{ rs34861192 } & $\mathrm{AA}(n=1)$ & 4.84 & 2.91 & 1.08 & 2.61 & 4.48 & 2.69 & 2.42 \\
\hline & $\mathrm{AG}(n=52)$ & $4.71 \pm 0.93$ & $1.97 \pm 1.32$ & $1.05 \pm 0.27$ & $2.75 \pm 0.77$ & $4.72 \pm 2.48$ & $2.13 \pm 1.79$ & $2.75 \pm 0.86$ \\
\hline & $\mathrm{GG}(n=146)$ & $4.43 \pm 0.91$ & $1.78 \pm 1.50$ & $1.07 \pm 0.27$ & $2.60 \pm 0.76$ & $4.30 \pm 1.15$ & $1.87 \pm 1.98$ & $2.52 \pm 0.81$ \\
\hline & $p$ & 0.156 & 0.551 & 0.073 & 0.587 & 0.090 & 0.653 & 0.670 \\
\hline \multirow[t]{4}{*}{ rs3219175 } & $\mathrm{AA}(n=1)$ & 4.84 & 2.91 & 1.08 & 2.61 & 4.48 & 2.69 & 2.42 \\
\hline & $\mathrm{AG}(n=50)$ & $4.75 \pm 0.93$ & $2.01 \pm 1.33$ & $1.05 \pm 0.28$ & $2.77 \pm 0.78$ & $4.76 \pm 1.23$ & $2.17 \pm 1.82$ & $2.77 \pm 0.87$ \\
\hline & $\mathrm{GG}(n=146)$ & $4.42 \pm 0.91$ & $1.78 \pm 1.50$ & $1.07 \pm 0.27$ & $2.59 \pm 0.76$ & $4.29 \pm 1.15$ & $1.87 \pm 1.98$ & $2.51 \pm 0.80$ \\
\hline & $p$ & 0.097 & 0.492 & 0.835 & 0.356 & 0.058 & 0.587 & 0.152 \\
\hline \multirow[t]{4}{*}{ rs3745368 } & $\mathrm{AA}(n=2)$ & $4.11 \pm 0.64$ & $1.13 \pm 0.01$ & $1.14 \pm 0.23$ & $2.20 \pm 0.80$ & $4.38 \pm 1.67$ & $1.18 \pm 0.26$ & $2.40 \pm 1.37$ \\
\hline & $\mathrm{AG}(n=41)$ & $4.37 \pm 0.95$ & $1.76 \pm 1.61$ & $1.04 \pm 0.22$ & $2.56 \pm 0.81$ & $4.35 \pm 1.23$ & $1.92 \pm 2.24$ & $2.55 \pm 0.88$ \\
\hline & $\mathrm{GG}(n=156)$ & $4.54 \pm 0.91$ & $1.87 \pm 1.42$ & $1.07 \pm 0.28$ & $2.66 \pm 0.76$ & $4.43 \pm 1.17$ & $1.96 \pm 1.86$ & $2.59 \pm 0.80$ \\
\hline & $p$ & 0.453 & 0.722 & 0.718 & 0.531 & 0.927 & 0.853 & 0.912 \\
\hline \multirow[t]{4}{*}{ rs1477341 } & $\mathrm{AA}(n=32)$ & $2.00 \pm 1.40$ & $4.41 \pm 0.82$ & $1.01 \pm 0.22$ & $2.50 \pm 0.71$ & $4.52 \pm 1.15$ & $2.17 \pm 1.69$ & $2.56 \pm 0.84$ \\
\hline & $\operatorname{AT}(n=90)$ & $1.96 \pm 1.47$ & $4.57 \pm 0.94$ & $1.04 \pm 0.25$ & $2.71 \pm 0.78$ & $4.58 \pm 1.16$ & $2.10 \pm 1.98$ & $2.70 \pm 0.79$ \\
\hline & $\Pi(n=68)$ & $1.62 \pm 1.53$ & $4.45 \pm 0.97$ & $1.13 \pm 0.30$ & $2.58 \pm 0.78$ & $4.12 \pm 1.19$ & $1.65 \pm 2.06$ & $2.39 \pm 0.84$ \\
\hline & $p$ & 0.302 & 0.588 & $0.049^{*}$ & 0.315 & $0.049^{*}$ & 0.292 & 0.066 \\
\hline
\end{tabular}

$p$ values were calculated by logistic regression

Italicized values are statistically significant

${ }^{*} p<0.05$ indicates statistical significance

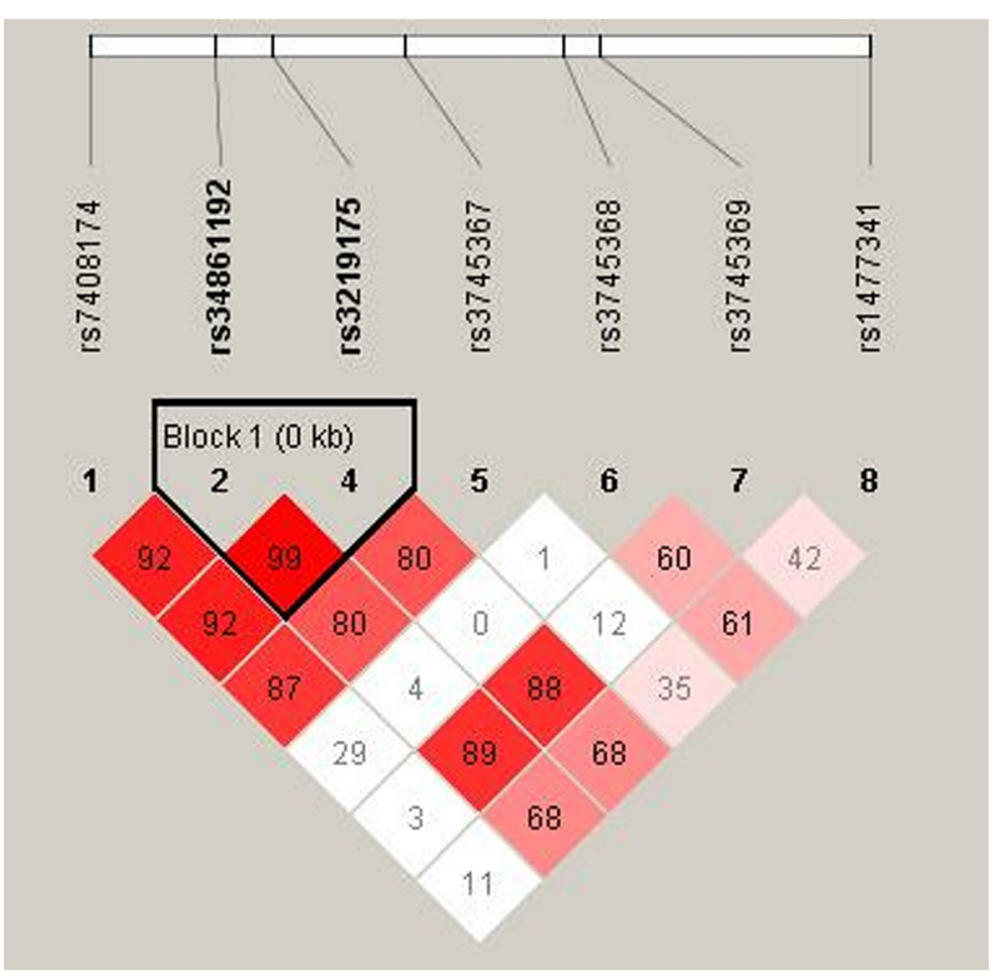

Fig. 1 Haplotype block map for the eight SNPs in the RETN gene. Block 1 includes rs34861192 and rs3219175 with $D^{\prime}=1$ (100\%) for the corresponding variants 


\section{Conclusions}

To sum up, the present study confirmed for the first time that the RETN polymorphisms rs34861192, rs3219175, rs3745368, and rs1477341 were associated with a reduced risk of steroid-induced ONFH, which may provide the basis for elucidating the pathogenesis of osteonecrosis.

\section{Abbreviations \\ 95\% Cl: 95\% confidence intervals; HWE: Hardy-Weinberg equilibrium; LD: Linkage disequilibrium; MAF: Minor allele frequency; OR: Odds ratio; SNP: Single-nucleotide polymorphism; steroid-induced ONFH: Steroid- induced osteonecrosis of the femoral head}

\section{Acknowledgements}

We are grateful to the individuals for their participation in this study. We also thank the clinicians and hospital staff who contributed to the sample and data collection for this study. We would also like to thank all participants in this manuscript.

\section{Authors' contributions}

FA and JianW conceived and designed the experiments. LZ wrote the article. $H G$ and JiaqW performed the experiments. $C L$ and $Y T$ analyzed the data. CM, $\mathrm{JZ}$, and KW contributed reagents/materials/analysis tools. All authors contributed significantly to the final draft of the paper and agreed to submit the manuscript for publication.

\section{Funding}

This study was funded by the National Natural Science Foundation of China (no. 81660228, no.81260284 and no.81660378).

\section{Availability of data and materials}

The datasets used or analyzed during the current study are available from the corresponding author on reasonable request.

\section{Ethics approval and consent to participate}

The use of human blood sample and the protocol in this study were strictly complied with the criterion of the Declaration of Helsinki and were approved by the Ethics Committee of the orthopedic hospital of Inner Mongolia Medical University (Inner Mongolia, China). Each participant signed the informed consent form after fully understanding the purpose of our study.

\section{Consent for publication}

Written informed consent was obtained from the patient for publication of this report.

\section{Competing interests}

The authors declare that they have no competing interests.

\section{Author details}

${ }^{1}$ Inner Mongolia Medical University, Hohhot 010050, Inner Mongolia, China. ${ }^{2}$ Department of Trauma Orthopedics, The Second Affiliated Hospital of Inner Mongolia Medical University, Hohhot 010030, Inner Mongolia, China. ${ }^{3}$ The Second Affiliated Hospital of Xi'an Jiaotong University, \#157 Xi Wu Road, Xi'an 710004, Shaanxi Province, China.

Received: 11 November 2019 Accepted: 14 January 2020 Published online: 06 March 2020

References

1. Du J, et al. Association between genetic polymorphisms of MMP8 and the risk of steroid-induced osteonecrosis of the femoral head in the population of northern China. Medicine (Baltimore). 2016;95(37):e4794.

2. Wang A, Ren M, Wang J. The pathogenesis of steroid-induced osteonecrosis of the femoral head: a systematic review of the literature. Gene. 2018; 10(671):103-9. https://doi.org/10.1016/j.gene.2018.05.091

3. Nakatochi M, et al. Epigenome-wide association study suggests that SNPs in the promoter region of RETN influence plasma resistin level via effects on DNA methylation at neighbouring sites. Diabetologia. 2015;58(12):2781-90.
4. Zhao X, et al. Glucocorticoid enhanced the expression of ski in osteonecrosis of femoral head: the effect on adipogenesis of rabbit BMSCs. Calcif Tissue Int. 2019;105(5):506-17.

5. Aimaiti A, et al. Can bisphenol A diglycidyl ether (BADGE) administration prevent steroid-induced femoral head osteonecrosis in the early stage? Med Hypotheses. 2011;77(2):282-5.

6. Zhang $Y$, et al. Vitamin K2 ameliorates damage of blood vessels by glucocorticoid: a potential mechanism for its protective effects in glucocorticoid-induced osteonecrosis of the femoral head in a rat model. Int J Biol Sci. 2016;12(7):776-85.

7. Du J, et al. A single-nucleotide polymorphism in MMP9 is associated with decreased risk of steroid-induced osteonecrosis of the femoral head. Oncotarget. 2016;7(42):68434-41.

8. Qi Y, et al. MMP-14 single-nucleotide polymorphisms are related to steroidinduced osteonecrosis of the femoral head in the population of northern China. Mol Genet Genomic Med. 2019;7(2):e00519. https://doi.org/10.1002/ mgg3.519.

9. Zhang Y, et al. Genetic association of the P-glycoprotein gene ABCB1 polymorphisms with the risk for steroid-induced osteonecrosis of the femoral head in Chinese population. Mol Biol Rep. 2014;41(5):3135-46.

10. Ma W, et al. Relationship of common variants in VEGFA gene with osteonecrosis of the femoral head: a Han Chinese population based association study. Sci Rep. 2018;8(1):16221

11. Kim HJ, et al. Expression of resistin in the prostate and its stimulatory effect on prostate cancer cell proliferation. BJU Int. 2011;108(2 Pt 2):E77-83.

12. Younis $\mathrm{S}$, Blumenberg $\mathrm{M}$, Javed $\mathrm{Q}$. Resistin gene polymorphisms are associated with acne and serum lipid levels, providing a potential nexus between lipid metabolism and inflammation. Arch Dermatol Res. 2016; 308(4):229-37.

13. Hornik B, et al. Physical activity increases the resistin concentration in hemodialyzed patients without metabolic syndrome. Diabetes Metab Syndr Obes. 2019;12:43-57.

14. Johnson C, et al. Increased expression of Resistin in MicroRNA-155-deficient white adipose tissues may be a possible driver of metabolically healthy obesity transition to classical obesity. Front Physiol. 2018:9:1297.

15. Ohmori R, et al. Associations between serum resistin levels and insulin resistance, inflammation, and coronary artery disease. J Am Coll Cardiol. 2005;46(2):379-80

16. Ikeda $Y$, et al. Resistin affects lipid metabolism during adipocyte maturation of 3T3-L1 cells. FEBS J. 2013;280(22):5884-95.

17. Gabriel S, Ziaugra L, Tabbaa D. SNP genotyping using the Sequenom MassARRAY iPLEX platform. Curr Protoc Hum Genet. 2009;Chapter 2:Unit 2.12.

18. Lehrke $M$, et al. An inflammatory cascade leading to hyperresistinemia in humans. PLoS Med. 2004;1(2):e45.

19. Reilly MP, et al. Resistin is an inflammatory marker of atherosclerosis in humans. Circulation. 2005;111(7):932-9.

20. McTernan $\mathrm{CL}$, et al. Resistin, central obesity, and type 2 diabetes. Lancet 2002;359(9300):46-7.

21. Degawa-Yamauchi $M$, et al. Serum resistin (FIZZ3) protein is increased in obese humans. J Clin Endocrinol Metab. 2003;88(11):5452-5.

22. Verma $\mathrm{S}$, et al. Resistin promotes endothelial cell activation: further evidence of adipokine-endothelial interaction. Circulation. 2003;108(6):736-40.

23. Onuma $\mathrm{H}$, et al. A at single nucleotide polymorphism-358 is required for $\mathrm{G}$ at -420 to confer the highest plasma resistin in the general Japanese population. PLoS One. 2010:5(3):e9718.

24. Asano $\mathrm{H}$, et al. Plasma resistin concentration determined by common variants in the resistin gene and associated with metabolic traits in an aged Japanese population. Diabetologia. 2010;53(2):234-46.

25. Apalasamy YD, et al. Polymorphisms of the resistin gene and their association with obesity and resistin levels in Malaysian Malays. Biochem Genet. 2015:53(4-6):120-31.

26. Hivert MF, et al. Association of variants in RETN with plasma resistin levels and diabetes-related traits in the Framingham offspring study. Diabetes. 2009;58(3):750-6.

27. Osawa $\mathrm{H}$, et al. Is rs34861192 or rs1862513 a more promising variant for determining plasma resistin in an aged Japanese population? Diabetologia. 2010;53(4):795-7.

\section{Publisher's Note}

Springer Nature remains neutral with regard to jurisdictional claims in published maps and institutional affiliations. 